\title{
Numerical simulation of heat transfer and fluid flow in coaxial laser cladding process for direct metal deposition
}

\author{
Huan Qi ${ }^{\text {a) }}$ and Jyotirmoy Mazumder \\ Center for Laser Aided Intelligent Manufacturing, Department of Mechanical Engineering, \\ The University of Michigan, Ann Arbor, Michigan 48109 \\ Hyungson $\mathrm{Ki}$ \\ Department of Mechanical Engineering, Michigan State University, East Lansing, Michigan 48824
}

(Received 17 January 2006; accepted 24 April 2006; published online 18 July 2006)

\begin{abstract}
The coaxial laser cladding process is the heart of direct metal deposition (DMD). Rapid materials processing, such as DMD, is steadily becoming a tool for synthesis of materials, as well as rapid manufacturing. Mathematical models to develop the fundamental understanding of the physical phenomena associated with the coaxial laser cladding process are essential to further develop the science base. A three-dimensional transient model was developed for a coaxial powder injection laser cladding process. Physical phenomena including heat transfer, melting and solidification phase changes, mass addition, and fluid flow in the melt pool, were modeled in a self-consistent manner. Interactions between the laser beam and the coaxial powder flow, including the attenuation of beam intensity and temperature rise of powder particles before reaching the melt pool were modeled with a simple heat balance equation. The level-set method was implemented to track the free surface movement of the melt pool, in a continuous laser cladding process. The governing equations were discretized using the finite volume approach. Temperature and fluid velocity were solved for in a coupled manner. Simulation results such as the melt pool width and length, and the height of solidified cladding track were compared with experimental results and found to be reasonably matched. () 2006 American Institute of Physics. [DOI: 10.1063/1.2209807]
\end{abstract}

\section{INTRODUCTION}

Laser cladding with powder injection technique has been widely used in many industrial applications such as surface coating, innovative alloying, rapid prototyping, direct metal deposition (DMD), and molds/dies repairing. ${ }^{1-4}$ Laser cladding is essentially a fusion and solidification process, which involves complicated interactions between the laser beam, metal powders, the base material (substrate), and processing gases. Typical physical phenomena in laser cladding include laser-powder interactions, heat transfer, fusion, fluid flow, and solidification. In the case with relatively high power laser, one can observe vaporization and plasma formation. There are approximately $12-14$ variables which strongly influence the characteristics of the clad part. These variables include the following: actual laser power, beam diameter (defocus distance), spatial distribution (mode) of the laser beam, shielding gas flow, powder delivery gas flow, travel speed, powder feed rate, material properties (absorptivity, melting point, thermal conductivity, etc.), powder characteristics (particle size distribution and particle shape), powder delivery method (side injection or concentric injection), height increment per layer, percentage overlap between tracks, and toolpath patterns. Some of these processing parameters are strongly coupled to each other. Optimization of these processing parameters is necessary to obtain the desired dimensional accuracy and material integrity of laser clad parts. Process optimization requires both theoretical and experi-

${ }^{a)}$ Electronic mail: qih@ research.ge.com mental understanding of the associated physical phenomena. Numerical modeling offers a cost-efficient way to better understand the related complex physics in a laser cladding process. It helps to reveal the effects and significance of each processing parameters on the desired characteristics of clad parts. Successful theoretical guidance enables intelligent closed-loop control in a high quality and high stability process, and saves time and cost in transfering the laser cladding technology from one system to another.

In the past decade, many analytical and numerical laser cladding models have been developed by researchers, revealing the process dependences on related parameters. The early models were mostly based on a heat transfer model with a necessary consideration of phase transformations, i.e., melting and solidification, but few incorporated fluid flow calculations due to the involved complexity. Picasso et al. ${ }^{5}$ proposed a simple but realistic model for laser cladding, incorporating the attenuation of laser power due to the absorption of powder flows as well as the dependency of the workpiece absorptivity with respect to the clad geometry. The melt pool shape was simply computed using a threedimensional (3D) analytical solution, which assumed a predeposited layer of powder on the substrate. Jouvard et al. ${ }^{6}$ studied the relation between the laser power and the clad mass through the calculation of energy distribution in laser cladding. They suggested a method to evaluate two power thresholds, of which the first one is the power required for substrate melting, and the second one is the threshold power when the coaxial powder is directly melted by the beam and is, therefore, a liquid when contacting the substrate. Hoadley 
and $\operatorname{Rappaz}^{7}$ developed a two-dimensional (2D) finite element model to get the quasistationary temperature field in the clad layer. They took into account the melting of powders in the liquid pool, and updated the shape and position of the liquid free surface conforming to the thermal field. However, in their model the height and length of the melt pool have to be predefined. Yevko et $a l^{8}{ }^{8}$ modeled the preplaced powder cladding process by solving a three-dimensional heat transfer equation numerically. Kaplan and Groboth ${ }^{9}$ modeled a 3D quasisteady laser cladding process with the consideration of laser-powder interactions. Their model is simplified with the assumption that the dimensions of the melt pool remain similar to pure surface melting in the absence of a clad layer. The cladding shape in their model is defined by parametrized functions. Toyserkani et al. ${ }^{10}$ developed a 3D transient finite element model with the consideration of laser power attenuation through blown powder stream. They used modified thermal conductivity to take into account the thermocapillary phenomena of the liquid metal without calculating the fluid flow. The clad geometry was obtained by continuously stacking tiny layers of deposited mass in the melt pool area. Zhao et al. ${ }^{11}$ proposed a quasi-steady-state laser cladding model using a 3D finite element method, which is similar to the model of Hoadley and Rappaz but focusing on the dilution control. Huang et al. ${ }^{12}$ recently developed a $3 \mathrm{D}$ transient laser cladding model in which fluid flow is fully solved with an energy equation in a coupled manner. A solid/liquid continuum model ${ }^{13}$ was used to model the melting and solidification phenomena. They used a simple indicative variable method to update the growth of the molten pool free surface without calculating accurate surface geometry for curvature dependent flow.

In coaxial powder injection laser cladding, the melt pool, under the irradiation of a laser beam, is free to deform due to surface tension and other forces. The growth and evolution of the liquid/gas (L/G) interface (i.e., free surface of melt pool) determine the quality of solidified geometry and surface roughness of the clad layer. In order to capture the interactive relations of the free surface geometry and the process physics, a precise method of free surface tracking is necessary. In a laser-generated melt pool, large gradients of temperature and surface tension exist, which are also the driving forces for fluid flow. Geometries of the L/G interface, the normal and curvature of the free surface, determine the surface capillary and thermocapillary forces, and thus the fluid flow in the melt pool. In addition, an accurate L/G interface enables the calculation of the incident angle of the laser beam when striking onto the hump clad surface and enables a more realistic geometry-dependent energy absorption profile. The level-set method, ${ }^{14}$ which has been successfully used in tracking complex free surface movements, ${ }^{15}$ was adopted in this study to track the motion of the $\mathrm{L} / \mathrm{G}$ interface.

The physical model and associated assumptions used in this study are described in the next section. Mathematical formulations and associated boundary conditions of each considered physical models are given thereafter, including

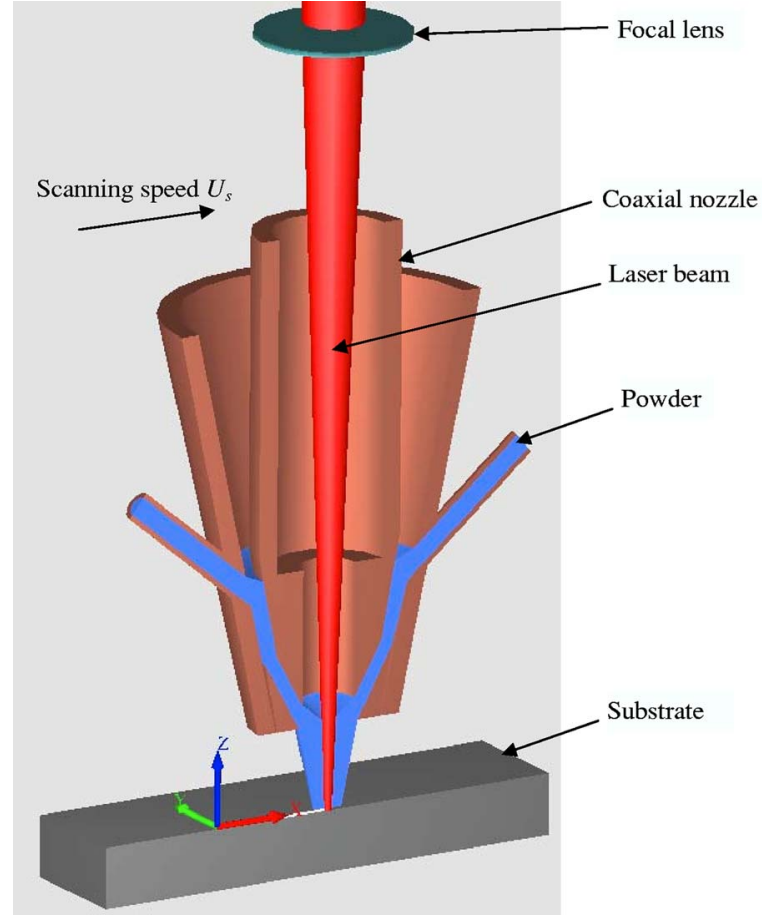

FIG. 1. (Color online) Diagram of laser cladding physical model.

heat transfer, melting/solidification phase change, mass addition, and liquid metal flow. The numerical and experimental results are compared in Sec. III.

\section{MATHEMATICAL MODELING}

\section{A. Physical model}

A schematic of the coaxial powder injection laser cladding process is shown in Fig. 1. In this physical model, the substrate is stationary in a three-dimensional Cartesian coordinate. A focused laser beam (propagating in the negative $Z$ direction) is striking on the substrate and moving at a constant speed $U_{s}$ in the positive $X$ direction. The origin is set on the top surface of the substrate and initially coincides with the center of the laser beam. The computational domain is initially composed of the substrate (solid phase) and the air (gas phase) above the substrate. Powders are delivered onto the substrate concentrically with the laser beam. The laser beam, when passing through the covered coaxial power stream, loses energy by reflection and absorption on powder particles. A small area of substrate surface under the irradiation of the laser beam gets heated and melted, and forms a molten pool. A clad track is formed (solidified from melt pool) with the scanning of the laser beam and continuous powder addition. The mathematical model in this study is based on the following assumptions.

- Laser beam profile is assumed to be Gaussian and constant along the $Z$ direction, i.e., the depth-of-focus effect is ignored.

- The liquid metal is considered an incompressible Newtonian fluid. Flow in the molten pool is considered to be laminar.

- The fluid flow is driven by the buoyancy force in the 


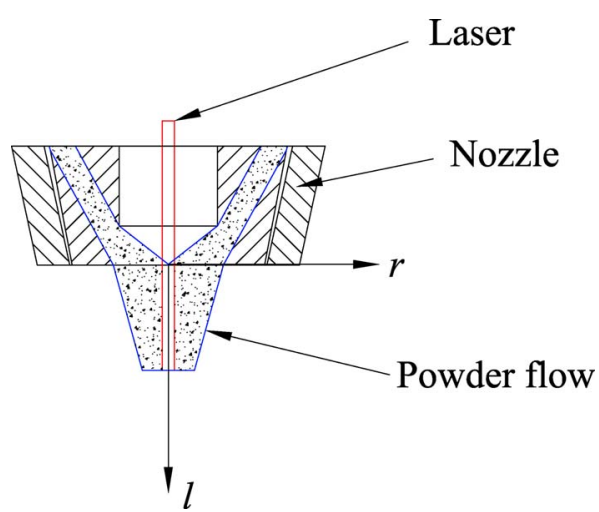

FIG. 2. (Color online) Laser-powder interaction through a coaxial nozzle.

molten pool, and the temperature and surface tension gradients on the molten pool surface.

- The solid and liquid phases are considered as a continuum medium. The velocity of solid phase is zero.

- The shape of the powder particle is spherical, and powders falling in the region of the liquid surface get melted immediately.

The last assumption was proposed based on a spherical particle melting model. ${ }^{16}$ The melting of a spherical particle bathed in the environment at constant melting temperature can be completed on the order of $10^{-4} \mathrm{~s}$ for a $100 \mu \mathrm{m}$ diameter steel particle, which is equivalent to one or two time steps in the numerical calculation of this model. Therefore, the melting of powder particle can be considered as instantaneous.

\section{B. Laser-powder interaction}

The laser beam when passing through the concentric powder stream from a coaxial nozzle (as shown in Fig. 2) gets attenuated by absorption, reflection, and scattering effects of the particle stream. Meanwhile, powder particles experience temperature rise and even phase changes (fusion and vaporization) before reaching the substrate. It has been verified experimentally that the spatial concentration profile of a converged coaxial powder flow can be approximated by a Gaussian distribution ${ }^{17}$ as defined in the following equation:

$$
N(r, l)=N_{\text {peak }}(l) \exp \left(\frac{-2 r^{2}}{R_{p}^{2}}\right),
$$

where $N$ is the number of powder particles in a unit volume and is a function of radial distance $r$ and axial distance $l$ in an axial-symmetrical coordinate, $N_{\text {peak }}$ is the peak concentration at the center of powder flow (where $r=0$ ), and $R_{p}$ is the effective radius of the powder stream at axial distance $l$. According to the Beer-Lambert law, the attenuation of laser beam intensity after passing through a distance $l$ in the powder flow can be expressed as

$$
q_{l}^{\prime}(r, l)=q_{l}(r) \exp \left(-\sigma_{\mathrm{ext}} N l\right),
$$

where $q_{l}^{\prime}(r, l)$ is the new (attenuated) laser power density, $q_{l}(r)$ is the original power density, and $\sigma_{\text {ext }}$ is the mean extinction area of powder particles. Laser power attenuation can be calculated step by step along the axial axis (from nozzle exit to the melt pool surface), where the attenuated laser power density of the upper layer is used as the incident power density of the adjacent lower layer.

The powder particles get heated when absorbing the laser energy. The temperature rise of the powder particles can be calculated with the following heat balance equation:

$$
q_{l}^{\prime}(r, l) \alpha_{p} \pi r_{p}^{2} \frac{\Delta l}{v_{p}}=\frac{4}{3} \pi r_{p}^{3} \rho_{p} C_{p}^{p} \Delta T,
$$

where $\alpha_{p}$ is the absorption coefficient of particles, $r_{p}$ is the radius of the particle, $v_{p}$ is the particle velocity, $\rho_{p}$ is the particle density, $C_{p}^{p}$ is the specific heat of the particle, and $\Delta T$ is the temperature rise of particles. The temperature rise of particles can also be calculated layer by layer along the axial axis using the attenuated laser power of each layer.

\section{Free surface tracking}

The level-set method was used in this model to track the evolution of the $\mathrm{L} / \mathrm{G}$ interface. In this method, we define a level-set function over the entire domain,

$$
\phi(\mathbf{x}, t)= \pm d,
$$

which is equal to the actual distance $d$ measured from the free surface of interest, where $\phi=0$. The plus (minus) sign denotes the outside (inside) of the interface. The definition of level-set function is schematically shown in Fig. 3. By using the level-set method the interface tracking problem can be transformed into a partial differential equation, which can be

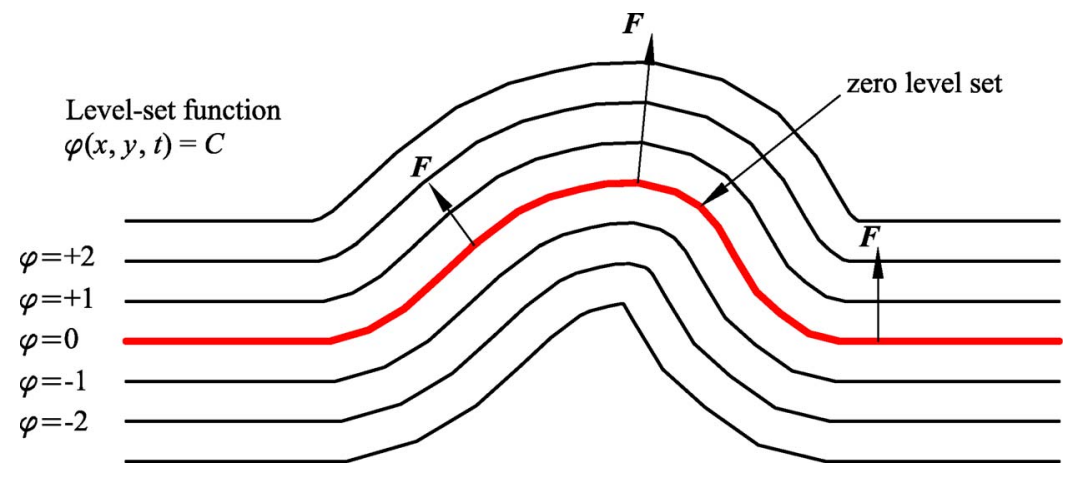

FIG. 3. (Color online) Definition of level-set function. 
numerically solved with other governing equations. The general form of the level-set equation is

$$
\frac{\partial \phi}{\partial t}+F|\nabla \phi|=0
$$

where $F$ is the force function (or speed function) that acts in the normal direction of the interface. In laser cladding, the motion of the liquid free surface $\mathrm{L} / \mathrm{G}$ interface) of the molten pool is caused by mass addition and local fluid flow, therefore, the force function can be defined as

$$
F=F_{p}+\mathbf{n} \cdot \mathbf{u}(x, y, z, t),
$$

where $F_{p}$ is the free surface growth velocity due to powder addition, $\mathbf{u}$ is the local fluid velocity, and $\mathbf{n}$ is the normal of the interface and is expressed as

$$
\mathbf{n}=\frac{\nabla \phi}{|\nabla \phi|}
$$

Here the powder addition speed $F_{p}$ can be formulated according to Eq. (1) as

$$
F_{p}=N_{s}(r) v_{p} \frac{4}{3} \pi r_{p}^{3}
$$

where $N_{s}(r)$ is the volume concentration profile of powder flow when reaching the substrate. The radial distance $r$ can also be expressed in the Cartesian coordinate as

$$
r=\sqrt{\left(x-U_{s} t\right)^{2}+y^{2}} \text {. }
$$

\section{Continuum model}

The computational domain contains all three phasessolid, liquid, and gas. In order to make the governing equations valid for the entire domain, the following schemes were employed in this study. The interface between the gas and nongas phases is considered physically sharp, and tracked by the level-set method as described in the previous section. The discrete gas and nongas phases were distinguished by the signs of the level-set function $\phi(\mathbf{x}, t)$. Material properties and appropriate calculation arrangement were made in different phases. However, the interface of solid and liquid phases in a multiconstituent alloying system can be morphologically very complex and forms a mushy zone that contains a mixture of both phases. In this study, the solid and liquid phases were treated as a continuum media, where the mushy region is a porous solid of isotropic permeability $K{ }^{18} \mathrm{~A}$ method developed by Bennon and Incropera ${ }^{13}$ was adopted to write the transport equations in a binary solid/liquid $(\mathrm{S} / \mathrm{L})$ phase change system. Defining mass fraction $f$ and volume fraction $g$, the velocity $\mathbf{u}$, density $\rho$, thermal conductivity $k$, specific heat $C_{p}$, mass diffusion coefficient $D$, solute concentration $c$, and enthalpy $h$ for a mixture of liquid and solid phases are

$$
\begin{aligned}
& \mathbf{u}=f_{l} \mathbf{u}_{l}, \\
& \rho=g_{s} \rho_{s}+g_{l} \rho_{l}, \\
& k=\left(\frac{g_{s}}{k_{s}}+\frac{g_{l}}{k_{l}}\right)^{-1},
\end{aligned}
$$

$$
\begin{aligned}
& C_{p}=f_{s} C_{p s}+f_{l} C_{p l}, \\
& D=f_{l} D_{l}, \\
& c=f_{s} c_{s}+f_{l} c_{l}, \\
& h=f_{s} h_{s}+f_{l} h_{l},
\end{aligned}
$$

respectively, where the subscripts $s$ and $l$ denote solid phase and liquid phase, respectively. Using the defined mixture variables, the energy equation and the momentum equations are given in the following sections.

\section{E. Heat transfer}

For a S/L binary phase system, the conservation of energy in enthalpy form ${ }^{13}$ can be written as

$$
\frac{\partial(\rho h)}{\partial t}+\nabla \cdot(\rho \mathbf{u} h)=\nabla \cdot(k \nabla T)-\nabla \cdot\left[\rho\left(h_{l}-h\right) \mathbf{u}\right] .
$$

Linearizing the phase enthalpies with the averaged specific heats $\bar{C}_{p l}, \bar{C}_{p s}$ and a constant $\mathcal{L}$ as defined in the following:

$$
\begin{aligned}
& h_{s}=\int_{0}^{T} C_{p s} d T=\bar{C}_{p s} T, \\
& h_{l}=\int_{0}^{T_{e}} C_{p s} d T+L_{m}+\int_{T_{e}}^{T} C_{p l} d T=\bar{C}_{p l} T+\mathcal{L}, \\
& \mathcal{L}=\left(\bar{C}_{p s}-\bar{C}_{p l}\right) T_{e}+L_{m}=\Delta \bar{C}_{p} T_{e}+L_{m},
\end{aligned}
$$

and using Eq. (16) we have

$$
h=\bar{C}_{p} T+f_{l} \mathcal{L},
$$

where $T_{e}$ is the eutectic temperature and $L_{m}$ is the latent heat of fusion. Replacing the enthalpies in Eq. (17) with Eqs. (18)-(21), we obtain the energy equation in temperature form as

$$
\frac{\partial\left(\rho C_{p} T\right)}{\partial t}+\mathbf{u} \cdot \nabla\left(\rho C_{p} T\right)=k \nabla^{2} T+S_{\mathrm{S} / \mathrm{L}}^{T},
$$

where the source term $S_{\mathrm{S} / \mathrm{L}}^{T}$ is

$$
S_{\mathrm{S} / \mathrm{L}}^{T}=\frac{\partial\left(\rho f_{s} \Delta \bar{C}_{p} T\right)}{\partial t}-\frac{\partial\left(\rho f_{l} \mathcal{L}\right)}{\partial t} .
$$

The source term represents the enthalpy changes caused by melting/solidification at the S/L interface, also called the Stefan condition.

Supplementary relationships are required to update the liquid fraction $f_{l}$ during the melting/solidification process. A method developed by Voller et al. ${ }^{19}$ was implemented to solve for $f_{l}$ in each time step with the assumption of local composition equilibrium at the $\mathrm{S} / \mathrm{L}$ interface. The relation of a mixture concentration $c$ and the corresponding liquidus temperature can be derived from the equilibrium phase diagram in Fig. 4, where straight liquidus and solidus lines are presumed, 


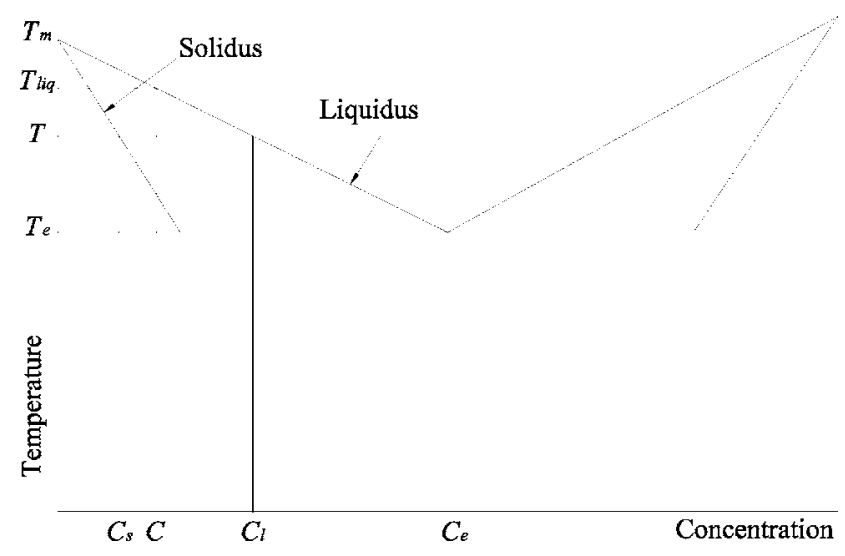

FIG. 4. Equilibrium phase diagram of a binary system.

$$
T_{\text {liq }}=T_{m}+\left(T_{e}-T_{m}\right) \frac{c}{c_{e}} .
$$

In Eq. (24), $T_{m}$ is the melting point and $c_{e}$ is the eutectic concentration. Using the lever rule and an equilibrium partition coefficient $k_{p}$, ${ }^{13}$ we have

$$
f_{l}=1-\frac{1}{1-k_{p}} \frac{T-T_{\mathrm{liq}}}{T-T_{m}} .
$$

From Eq. (25), the local temperature $T$ can be expressed as a function of $f_{l}$ and $T_{\text {liq. }}$. By equating the enthalpies at two sequential time steps $n$ and $n+1$, and replacing $T^{n+1}$ with Eq. (25), we can solve for $f_{l}^{n+1}$ using the following equation:

$$
\bar{C}_{p} T\left(f_{l}^{n+1}, c^{n}\right)+\mathcal{L} f_{l}^{n+1}=\bar{C}_{p} T^{n}+\mathcal{L} f_{l}^{n} .
$$

The boundary conditions at the $\mathrm{L} / \mathrm{G}$ interface include the energy gains, such as absorbed laser power and the heated powder entering the molten pool, and the energy losses such as convection and radiation heat transfers. A Gaussian distribution was considered for the laser beam

$$
q_{l}(r)=\frac{2 P}{\pi R_{b}^{2}} \exp \left(\frac{-2 r^{2}}{R_{b}^{2}}\right)
$$

where $r$ is defined in Eq. (9), $P$ is the total laser power, and $R_{b}$ is the effective laser beam radius. The actual laser power absorbed by the workpiece is a function of the material absorptivity $\alpha$ and the laser beam incident angle $\theta$. An absorption coefficient is defined in an empirical equation as

$$
A_{a}=\alpha|\cos (\theta)|^{0.2} \text {. }
$$

The extra energy brought by the addition of the heated powder (assuming the powder has the same material properties as the substrate) can be expressed as

$$
q_{p}= \begin{cases}F_{p} \rho_{l}\left[C_{p s}\left(T_{m}-T_{0}\right)+L_{m}+C_{p l}\left(T-T_{m}\right)\right], & T>T_{m}, \\ F_{p} \rho_{l}\left[C_{p s}\left(T_{m}-T_{0}\right)+L_{m} f_{l}-f_{s} L_{m}\right], & T=T_{m}, \\ F_{p} \rho_{s}\left[C_{p s}\left(T-T_{0}\right)-C_{p s}\left(T_{m}-T\right)-L_{m}\right], & T<T_{m} .\end{cases}
$$

The boundary conditions at the $\mathrm{L} / \mathrm{G}$ interface for the energy equation can be formulated as

$$
q_{\mathrm{L} / \mathrm{G}}=q_{l}(r) A_{a}+q_{p}-A_{h}\left(T-T_{0}\right)-\sigma \varepsilon\left(T^{4}-T_{0}^{4}\right),
$$

where $A_{h}$ denotes the heat transfer coefficient of the forced convection, $T_{0}$ is the ambient temperature, and $\sigma$ and $\varepsilon$ denote the Stefan-Boltzmann constant and the emissivity, respectively.

The boundary conditions in Eq. (30) can be added as a source term in Eq. (22) by multiplying with a delta function $\delta(\phi)$, where $\phi$ is the level-set value. The delta function $\delta(\phi)$ is defined as

$$
\delta(\phi)= \begin{cases}1, & \phi=0 \\ 0, & \phi \neq 0 .\end{cases}
$$

\section{F. Fluid flow}

The momentum equation in the $X$ direction for a binary $\mathrm{S} / \mathrm{L}$ system $^{13}$ can be written as

$$
\frac{\partial(\rho u)}{\partial t}+\nabla \cdot(\rho \mathbf{u} u)=\nabla \cdot\left(\mu_{l} \frac{\rho}{\rho_{l}} \nabla u\right)-\frac{\partial p}{\partial x}+S_{\mathrm{S} / \mathrm{L}}^{u},
$$

where $\mu_{l}$ is the liquid viscosity. The source term $S_{\mathrm{S} / \mathrm{L}}^{u}$ can be expressed as

$$
S_{\mathrm{S} / \mathrm{L}}^{u}=-\frac{\mu_{l}}{K} \frac{\rho}{\rho_{l}} u+\rho_{r} g_{x} \beta\left(T-T_{r}\right),
$$

where the first term is a Darcy term, representing the damping force when fluid passes through a porous media (dendrite structures). The isotropic permeability $K$ is assumed to vary with liquid volume fraction according to the Kozeny-Carman equation: ${ }^{20}$

$$
K=K_{0} \frac{g_{l}^{3}}{\left(1-g_{l}\right)^{2}},
$$

where $K_{0}$ is an empirical constant determined by the morphology of the porous media. $K \rightarrow 0$ corresponds to a complete solid phase, whereas the Darcy term dominates the momentum equation; $K \rightarrow \infty$ corresponds to a complete liquid phase, whereas the Darcy term disappears from the momentum equation. The second term in Eq. (33) is the buoyancy force caused by the temperature-dependent density gradient in the liquid phase (a Boussinesq model). In Eq. (33), $\rho_{r}$ is the liquid density under a reference temperature $T_{r}, \beta$ is the thermal expansion coefficient, and $g_{x}$ is the gravity component in the $X$ direction.

Two forces were considered at the $\mathrm{L} / \mathrm{G}$ interface-the capillary and thermocapillary forces. The capillary force acts in the normal direction due to the interface curvature and surface tension. The thermocapillary force acts in the tangential direction of the free surface and is caused by the surface temperature gradient. These forces can be formulated as

$$
\mathbf{F}_{\mathrm{L} / \mathrm{G}}=\sigma \mathbf{n}^{*} \kappa-\nabla_{s} T \frac{d \sigma}{d T}
$$

where $\sigma$ is the surface tension, $\mathbf{n}^{*}$ is the normal of the surface pointing inward to the liquid phase, and $\kappa$ is the curvature. Again, these surface force terms can be incorporated into the momentum equation as a source term by multiplying with a delta function $\delta(\phi)$. The final form of the momentum equa- 


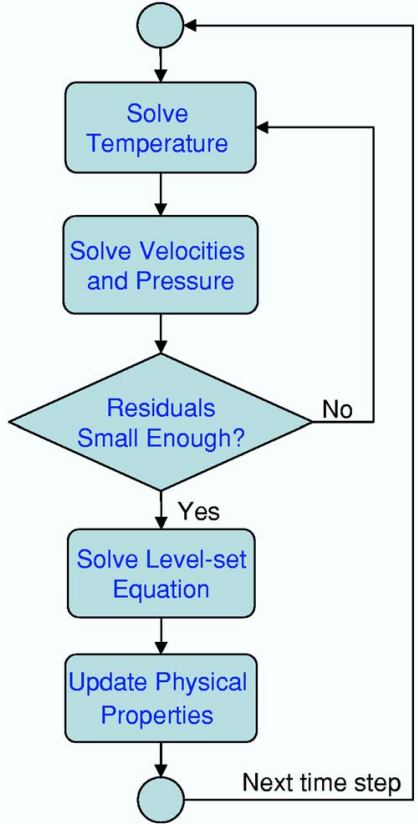

FIG. 5. (Color online) Flowchart of the solution procedure in each time step.

tions in the $X, Y$, and $Z$ directions can be formulated as follows:

$$
\begin{aligned}
\frac{\partial(\rho u)}{\partial t}+\nabla \cdot(\rho \mathbf{u} u)= & \nabla \cdot\left(\mu_{l} \frac{\rho}{\rho_{l}} \nabla u\right)-\frac{\partial p}{\partial x}-\frac{\mu_{l}}{K} \frac{\rho}{\rho_{l}} u \\
& -\mathbf{e}_{x} \cdot\left(\sigma \mathbf{n}^{*} \kappa-\nabla_{s} T \frac{d \sigma}{d T}\right) \delta(\phi), \\
\frac{\partial(\rho v)}{\partial t}+\nabla \cdot(\rho \mathbf{u} v)= & \nabla \cdot\left(\mu_{l} \frac{\rho}{\rho_{l}} \nabla v\right)-\frac{\partial p}{\partial y}-\frac{\mu_{l}}{K} \frac{\rho}{\rho_{l}} v \\
& -\mathbf{e}_{y} \cdot\left(\sigma \mathbf{n}^{*} \kappa-\nabla_{s} T \frac{d \sigma}{d T}\right) \delta(\phi),
\end{aligned}
$$

TABLE I. Materials properties for substrate and powder steel.

\begin{tabular}{lllr}
\hline \hline \multicolumn{1}{c}{ Property } & Symbol & \multicolumn{2}{c}{ Value } \\
\hline Melting temperature & $T_{m}$ & 1809.0 & $(\mathrm{~K})$ \\
Eutectic temperature & $T_{e}$ & 1776.0 & $(\mathrm{~K})$ \\
Boiling temperature & $T_{b}$ & 3133.0 & $(\mathrm{~K})$ \\
Liquid density & $\rho_{l}$ & 6518.5 & $\left(\mathrm{~kg} / \mathrm{m}^{3}\right)$ \\
Solid density & $\rho_{s}$ & 7870.0 & $\left(\mathrm{~kg} / \mathrm{m}^{3}\right)$ \\
Liquid viscosity & $\mu_{l}$ & 0.0050 & $\left(\mathrm{~N} \mathrm{~s} / \mathrm{m}^{2}\right)$ \\
Latent heat of fusion & $L_{m}$ & $2.7196 \times 10^{5}$ & $(\mathrm{~J} / \mathrm{kg})$ \\
Solid thermal conductivity & $K_{s}$ & 40.96 & $(\mathrm{~W} / \mathrm{m} \mathrm{K})$ \\
Liquid thermal conductivity & $K_{l}$ & 43.99 & $(\mathrm{~W} / \mathrm{m} \mathrm{K})$ \\
Thermal expansion coefficient & $\beta$ & $1.45 \times 10^{-4}$ & $(1 / \mathrm{K})$ \\
Solid specific heat & $C_{p s}$ & 658.63 & $(\mathrm{~J} / \mathrm{kg} \mathrm{K})$ \\
Liquid specific heat & $C_{p l}$ & 804.03 & $(\mathrm{~J} / \mathrm{kg} \mathrm{K})$ \\
Surface absorptivity for $\mathrm{CO}_{2}$ beam & $\alpha$ & 0.1 & \\
Surface tension coefficient & $\sigma_{T}$ & -0.00049 & $(\mathrm{~N} / \mathrm{m} \mathrm{K})$ \\
Darcy coefficient & $K_{0}$ & $1.0 \times 10^{-10}$ & $\left(\mathrm{~m}^{2}\right)$ \\
Mass diffusivity & $D_{l}$ & $3.0 \times 10^{-18}$ & $\left(\mathrm{~m}^{2} / \mathrm{s}\right)$ \\
\hline \hline
\end{tabular}

TABLE II. Processing parameters.

\begin{tabular}{lcr}
\hline \multicolumn{1}{c}{ Parameter } & \multicolumn{2}{c}{ Value } \\
\hline Laser power & $400-600$ & $(\mathrm{~W})$ \\
Powder flow rate & 6.0 & $(\mathrm{~g} / \mathrm{min})$ \\
Laser scanning speed & 12.7 & $(\mathrm{~mm} / \mathrm{s})$ \\
Powder jet radius at nozzle & 2.64 & $(\mathrm{~mm})$ \\
Powder jet radius at substrate & 1.50 & $(\mathrm{~mm})$ \\
Powder travel distance & 7 & $(\mathrm{~mm})$ \\
Powder flow velocity & 1.0 & $(\mathrm{~m} / \mathrm{s})$ \\
Powder particle radius & 0.075 & $(\mathrm{~mm})$ \\
Eutectic solute concentration & 0.5 & \\
Substrate solute concentration & 0.001 & \\
Powder solute concentration & 0.3 & \\
Equilibrium partition coefficient & \multicolumn{3}{c}{0.2} & \\
\hline \hline
\end{tabular}

$$
\begin{aligned}
\frac{\partial(\rho w)}{\partial t}+\nabla \cdot(\rho \mathbf{u} w)= & \nabla \cdot\left(\mu_{l} \frac{\rho}{\rho_{l}} \nabla w\right)-\frac{\partial p}{\partial z}-\frac{\mu_{l}}{K} \frac{\rho}{\rho_{l}} w \\
& +\rho_{r} g_{z} \beta\left(T-T_{r}\right)-\mathbf{e}_{z} \\
& \cdot\left(\sigma \mathbf{n}^{*} \kappa-\nabla_{s} T \frac{d \sigma}{d T}\right) \delta(\phi),
\end{aligned}
$$

where $\mathbf{e}_{x}, \mathbf{e}_{y}$, and $\mathbf{e}_{z}$ are the unit vectors in the $X, Y$, and $Z$ directions, respectively.

\section{G. Solutions}

In order to accurately calculate the strong fluid flow and the free surface motion of the molten pool, the grid size at the laser irradiated area have to be adequately small. For instance, the minimum grid size in the $X-Y$ plane has to be less than $50 \mu \mathrm{m}$ when ten or more cells are needed within the laser spot size. The minimum grid size in the $Z$ direction is set at $20 \mu \mathrm{m}$ in order to capture a cladding layer height within the range of $60-300 \mu \mathrm{m}$. It is assumed that the domain is symmetrical in the $Y$ direction, therefore only half of the domain is considered. In this study, the overall computational domain size is $13 \times 5 \times 10 \mathrm{~mm}$ in $X Y$, and $Z$, corre-

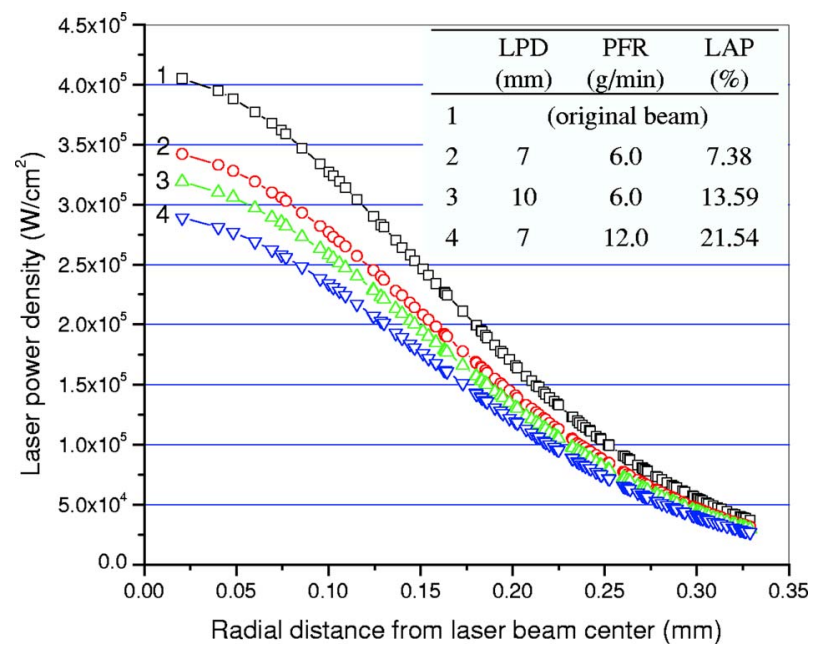

FIG. 6. (Color online) Attenuation of laser power $(500 \mathrm{~W})$ intensity after passing through powder flow with different parameters. LPD: laser-powder interaction distance, PFR: powder flow rate, and LAP: laser attenuation percentage. 


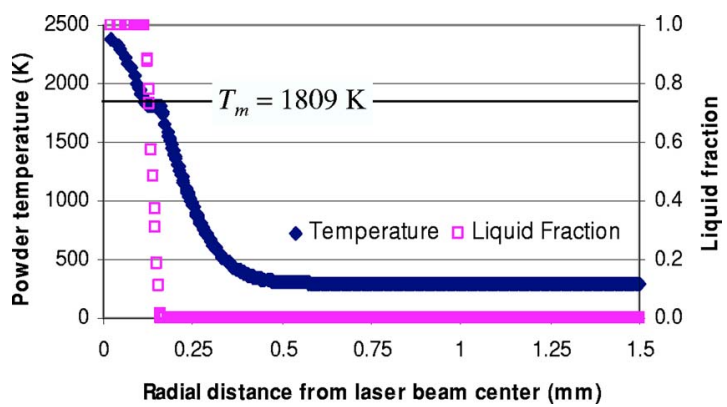

FIG. 7. (Color online) Temperature distribution and liquid fracture of powder flow when reaching the substrate surface, with the parameters laser power $(500 \mathrm{~W})$, powder flow rate $(6.0 \mathrm{~g} / \mathrm{min})$, travel distance $(7 \mathrm{~mm})$, and travel speed $(1 \mathrm{~m} / \mathrm{s})$

sponding to a nonuniform grid system of $93 \times 37 \times 53$. The boundaries of the computational domain is considered far enough from the melt pool region so that infinite boundary conditions are employed.

The energy equation was discretized with an implicit finite difference method and solved for the entire threedimensional domain. The advection of dependent variables was formulated with an upwind scheme. A semi-implicit method for pressure-linked equations consistent (SIMPLEC) method $^{21}$ was used to solve the coupled pressure-velocity

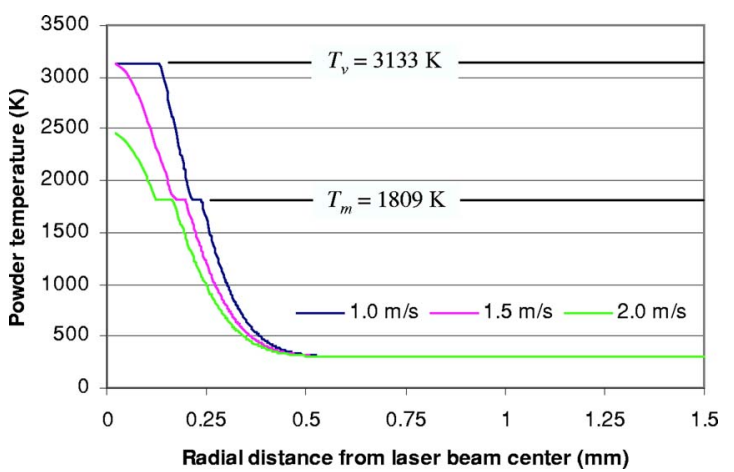

FIG. 8. (Color online) Temperature distribution of powder flow when reaching the substrate surface, with the parameters laser power $(1000 \mathrm{~W})$, powder flow rate $(6.0 \mathrm{~g} / \mathrm{min})$, travel distance $(7 \mathrm{~mm})$, and travel speed $(1.0,1.5$, and $2.0 \mathrm{~m} / \mathrm{s})$.

fields on a staggered grid system. The solving variables were all underrelaxed to avoid unpredicted divergence. A relaxation factor of 0.7 was found to be adequate for $u, v, w$, and $T$ equations. The level-set equation was solved point by point with an explicit method. The time step of this problem was adapted according to the strength of the fluid flow in the molten pool. It is restricted to be no more than $10^{-3} \mathrm{~s}$ to meet the convergence criteria of the numerical solution and the
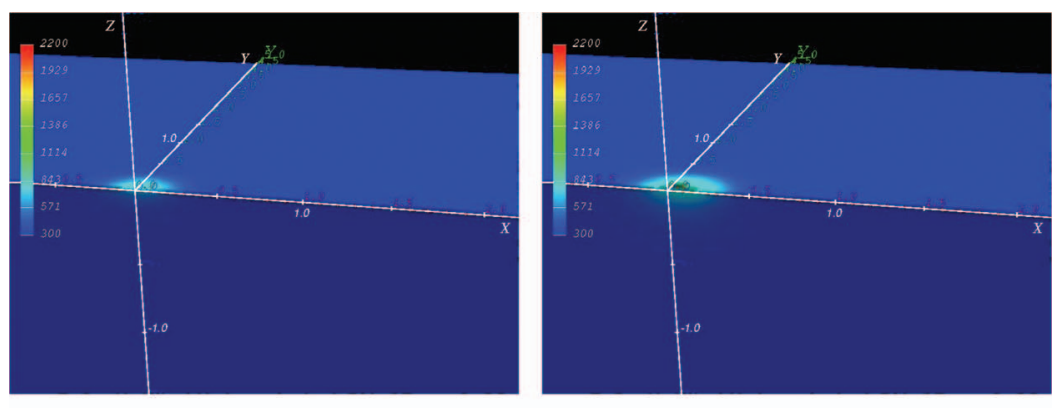

(a) $\mathrm{t}=3.01 \mathrm{~ms}$
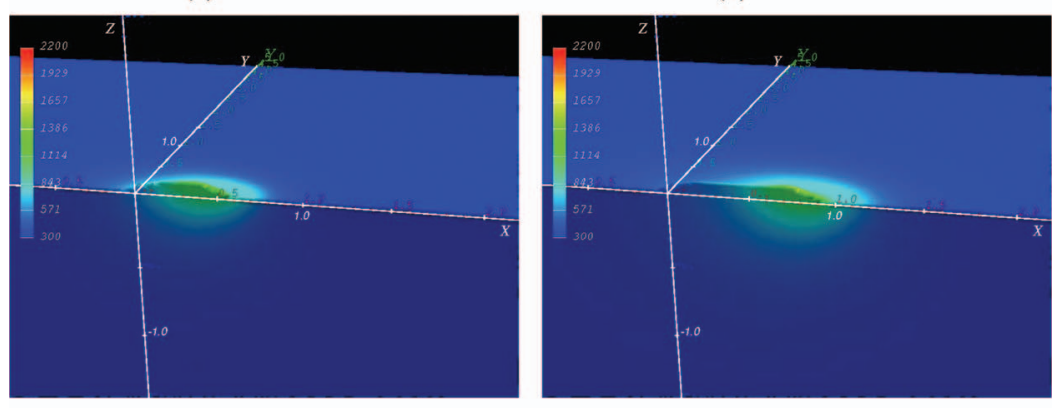

(c) $\mathbf{t}=38.21 \mathrm{~ms}$

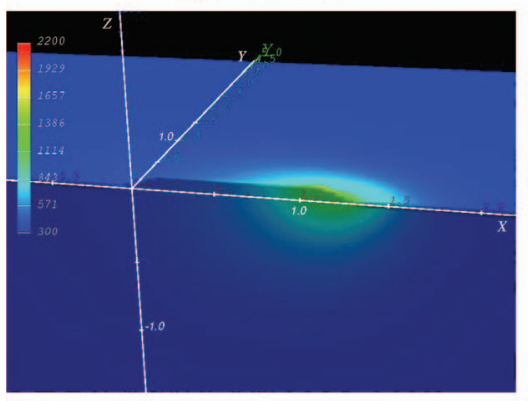

(e) $\mathrm{t}=95.81 \mathrm{~ms}$ (d) $\mathbf{t}=66.21 \mathrm{~ms}$

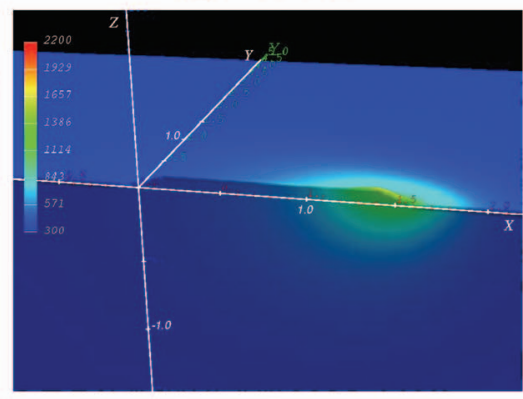

(f) $\mathrm{t}=119.00 \mathrm{~ms}$
FIG. 9. (Color) Sequential temperature fields as in the evolution of a continuous cladding track with a laser power of $400 \mathrm{~W}$. 

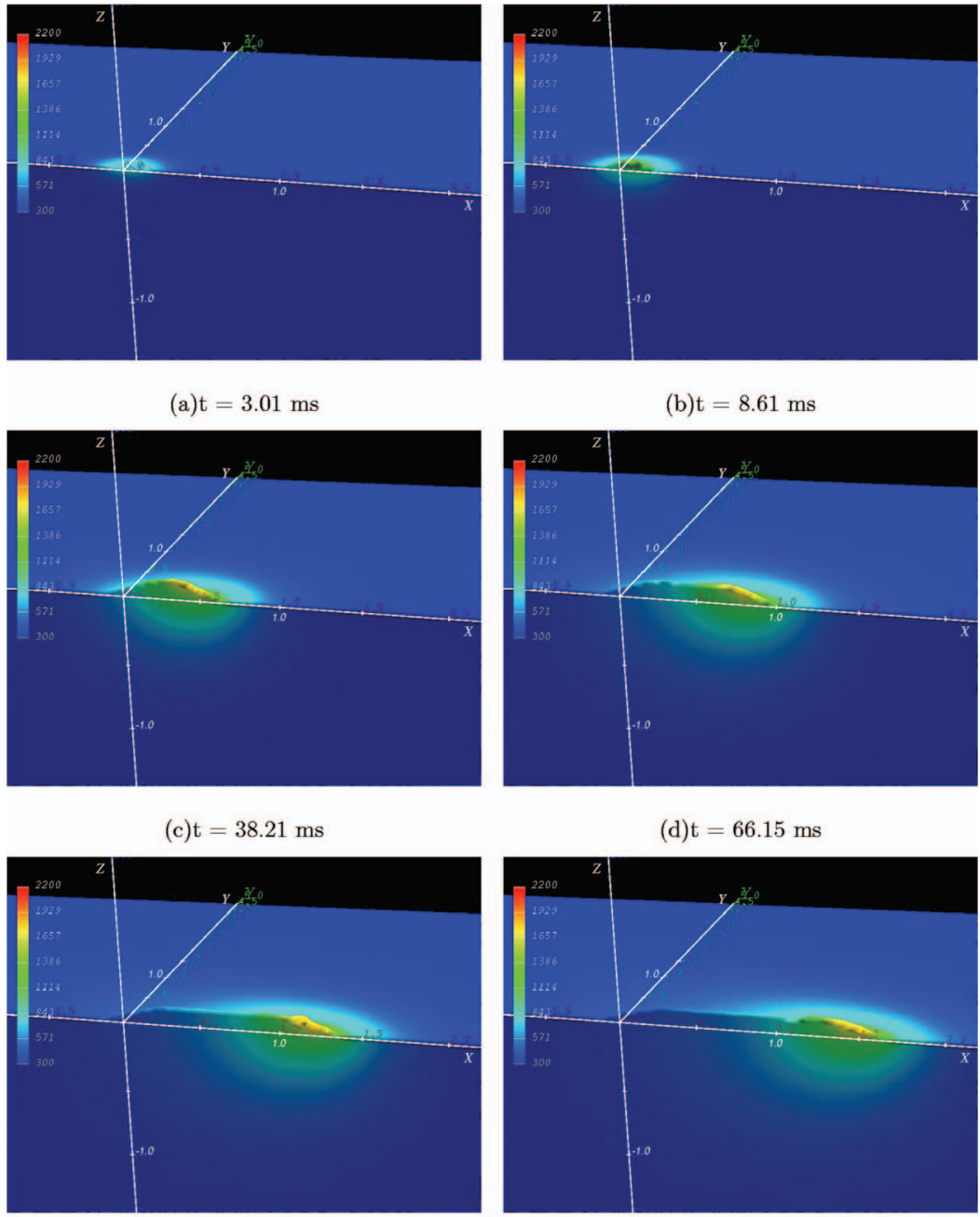

$(\mathrm{e}) \mathrm{t}=96.01 \mathrm{~ms}$

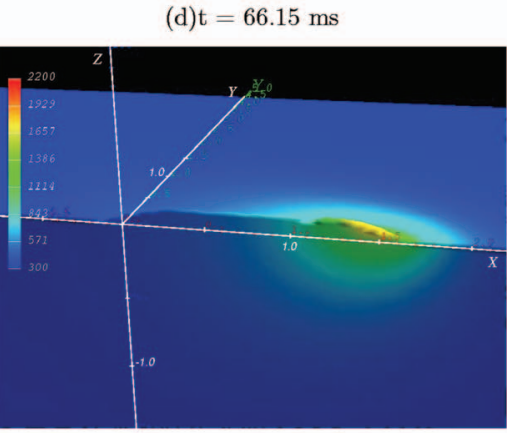

(f) $\mathrm{t}=119.20 \mathrm{~ms}$
FIG. 10. (Color) Sequential temperature fields as in the evolution of a continuous cladding track with a laser power of $500 \mathrm{~W}$. motion speed of the free surface. The maximum displacement of the free surface at one time step should be less than the minimum grid spacing.

The calculation scheme in every time step is as follows:

- The energy equation was first solved according to the updated physical properties and the L/G interface geometry from the last time step.

- The velocities and pressure were solved in a coupled manner.

- The residuals of calculated variables were checked. If they do not satisfy the convergence criteria, the program goes back to the first step; otherwise, continue.

- The level-set equation was solved, and the L/G interface was updated.

- Physical properties and liquid fractions were updated.

- Adaptive time step size was calculated for the next time step.

Figure 5 shows the flowchart of the solution procedure in one time step. Note that the temperature and velocities are solved sequentially in an inner loop for coupled solutions. A maximum iteration is set to prevent unnecessary computations of slow convergence, even though the residual values are not reached. This normally works well with a tight time step.

\section{NUMERICAL RESULTS}

In this study, we considered a continuous wave $\mathrm{CO}_{2}$ laser beam with a Gaussian distribution and a $600 \mu \mathrm{m}$ beam diameter. The substrate material is low-carbon steel. Material properties are given in Table. I. ${ }^{26}$ The same material properties were considered for powder particles. The processing parameters are given in Table II.

\section{A. Laser-powder interaction}

With a $500 \mathrm{~W}$ original incident laser beam, the numerical results of the laser power attenuation and the powder temperature rise when reaching the molten pool free surface are shown in Figs. 6 and 7, respectively. Figure 6 demonstrates that by increasing the powder flow rate or the laserpowder interaction distance (i.e., nozzle standoff distance), the attenuation of laser power increases. The total attenuated laser powers, in this parametric study, are in the range of $7 \%-21 \%$ of the original laser power with a $6-12 \mathrm{~g} / \mathrm{min}$ powder flow rate and a 7-10 mm interaction distance. A comparable experimental result was reported in Ref. 22 where $11 \%$ of the total $\mathrm{CO}_{2}$ laser beam was found to be reflected and absorbed by a lateral-injected steel powder stream. The temperature and the liquid fraction distribution of the powder flow at $6 \mathrm{~g} / \mathrm{min}$ after traveling $7 \mathrm{~mm}$ at the 

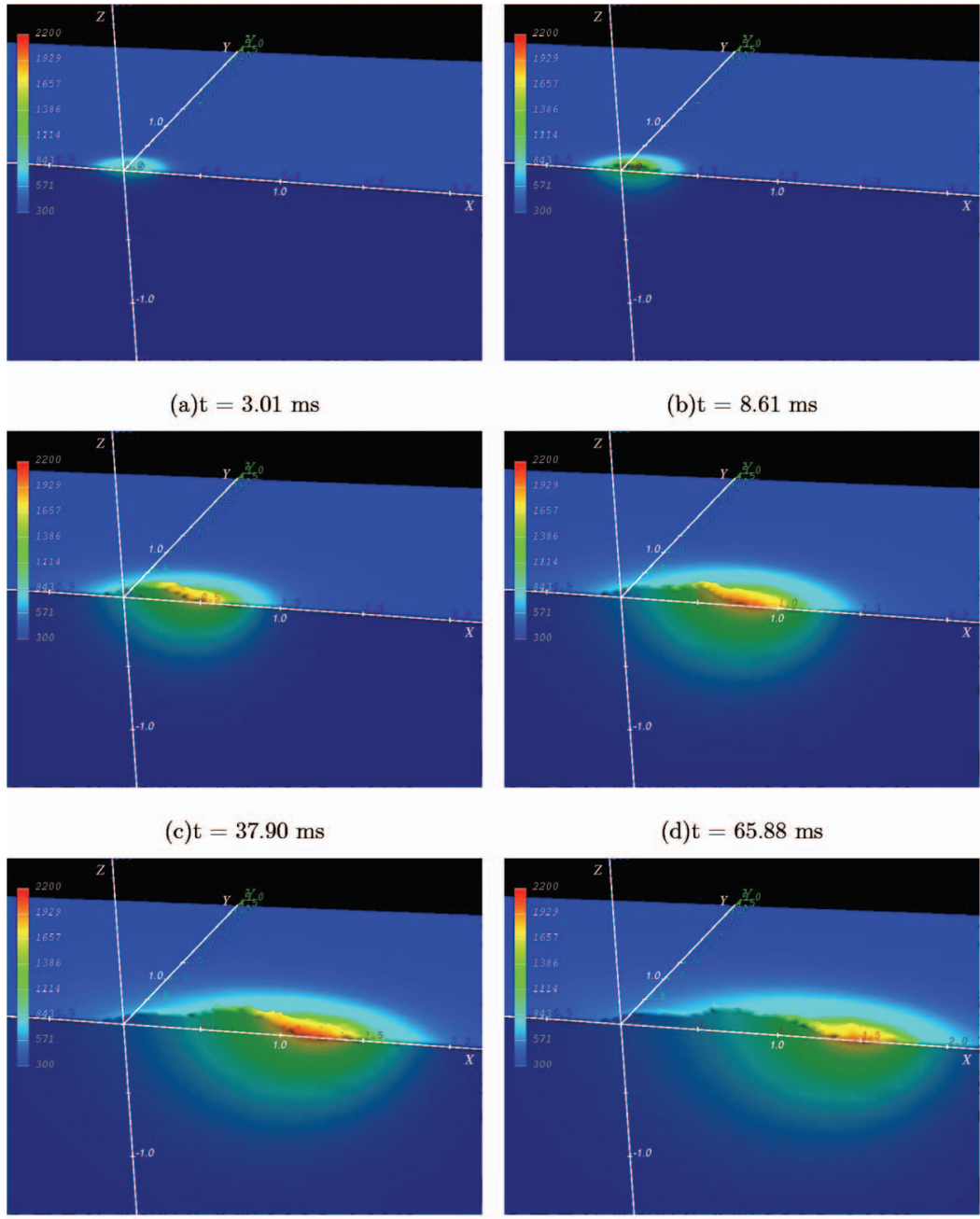

$(\mathrm{e}) \mathrm{t}=95.81 \mathrm{~ms}$

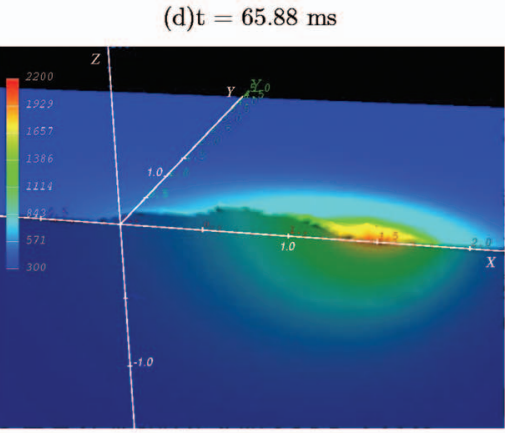

(f) $\mathrm{t}=119.40 \mathrm{~ms}$
FIG. 11. (Color) Sequential temperature fields as in the evolution of a continuous cladding track with a laser power of $600 \mathrm{~W}$. speed of $1 \mathrm{~m} / \mathrm{s}$ (Ref. 23) are plotted in Fig. 7. It can be seen from Fig. 7, that a portion of the powder flow (within the radius of $0.2 \mathrm{~mm}$ ) is already in the molten status when reaching the melt pool. Figure 8 shows the powder temperature distribution with a laser power of $1000 \mathrm{~W}$ and different powder flow velocities at $1.0,1.5$, and $2.0 \mathrm{~m} / \mathrm{s}$, while the other parameters were kept the same. Note that the vaporization temperature $(3133 \mathrm{~K})$ is reached for powder velocities of less than $1.5 \mathrm{~m} / \mathrm{s}$. With a $1.0 \mathrm{~m} / \mathrm{s}$ velocity, the flying powder particles within a radius of $0.13 \mathrm{~mm}$ begin vaporizing upon reaching the melt pool. To maintain powder capture efficiency and avoid undesired plasma formation, the vaporization of the powder should be avoided.

\section{B. Temperature and velocity fields}

Figures 9-11 demonstrate the sequential temperature fields of a clad track generated in a $120 \mathrm{~ms}$ period with the laser power settings of 400,500, and $600 \mathrm{~W}$, respectively. At $400 \mathrm{~W}$, only the melted powders are deposited on the cold substrate and solidified instantly. A quasi-steady-state heat balance is soon reached after about $30 \mathrm{~ms}$. The input energy is not enough to cause a melt pool on the substrate in this case. The highest temperature obtained is $1553.2 \mathrm{~K}$. Because there is no fluid flow in the cladding layer, the shape of the clad track is very smooth and uniform in the simulation result. In the $500 \mathrm{~W}$ case (Fig. 10), a very small molten pool is formed, but is almost limited within the cladding layer. The maximum temperature in the melt pool is $1861.1 \mathrm{~K}$. There is very little melting in the substrate. In this case the clad layer has minimum dilution in the substrate. When the laser power increases to $600 \mathrm{~W}$ (Fig. 11), a $0.5 \mathrm{~mm}$ long and $0.3 \mathrm{~mm}$ wide molten pool is formed. It can be observed that the molten pool size keeps increasing until the laser beam travels to $X \approx 1.0 \mathrm{~mm}$. Temperature as high as $2001.6 \mathrm{~K}$ is reached in the melt pool. Because of the large surface temperature gradient, the liquid in the molten pool flows outwards from the center area (see Fig. 12). This motion, combined with the scanning speed, "piles up" the continuously added material behind the molten pool and subsides the molten pool surface. The strong fluid flow inside the molten pool causes the formation of a fluctuating cladding surface. The substrate starts melting as the laser beam moves to $0.5 \mathrm{~mm}$ in the $X$ direction. Figure 12 demonstrates the sequential velocity fields of the melt pool during the formation of the clad track with a laser power of $600 \mathrm{~W}$. The maximum velocity magnitude is $1.12 \mathrm{~m} / \mathrm{s}$ when the molten pool is fully developed, as shown in Fig. 12(d). A thin layer of substrate gets melted after the 

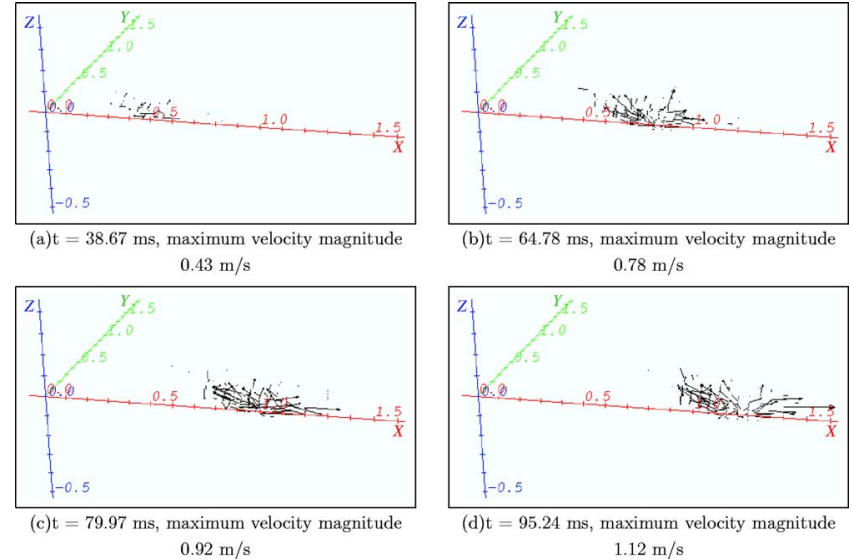

FIG. 12. Sequential velocity fields of the melt pool as in the evolution of a continuous cladding track with a laser power of $600 \mathrm{~W}$.

molten pool is fully developed, which makes the fluid flow extend into the substrate. A good metallurgical bonding can be formed in this case.

\section{Comparisons to experimental results}

To compare with the numerical results, the same processing parameters were used in a cladding experiment, in which AISI H13 tool steel powder was deposited on a carbon steel substrate. A schematic diagram of the experimental setup is demonstrated in Fig. 13. During the laser cladding process, an argon-ion laser beam is used to illuminate the molten pool. The reflected images are filtered through a narrow bandpass filter attached on a high speed camera, which only allows a narrow transmission band of light at the wavelength of the argon-ion laser $(515.5 \pm 5 \mathrm{~nm})$ to pass through and be recorded by the high speed camera. In this way, the reflected high brightness of $\mathrm{CO}_{2}$ beam from the cladding zone was shielded off. The argon-ion laser was delivered to the cladding workstation via an optical fiber, then focused by

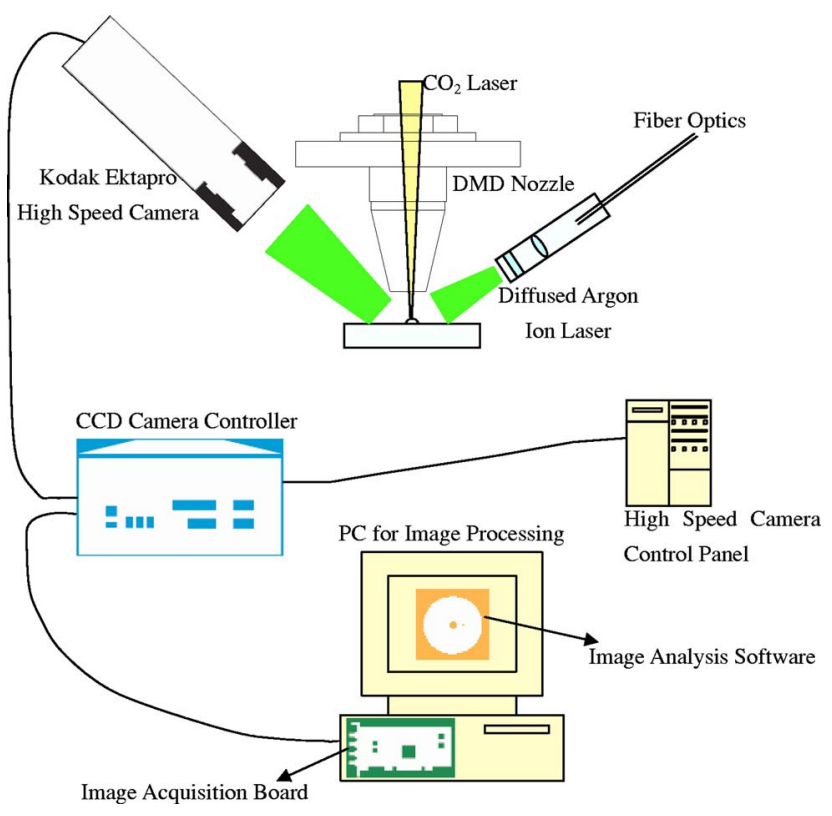

FIG. 13. Experimental setup of laser cladding visualization.

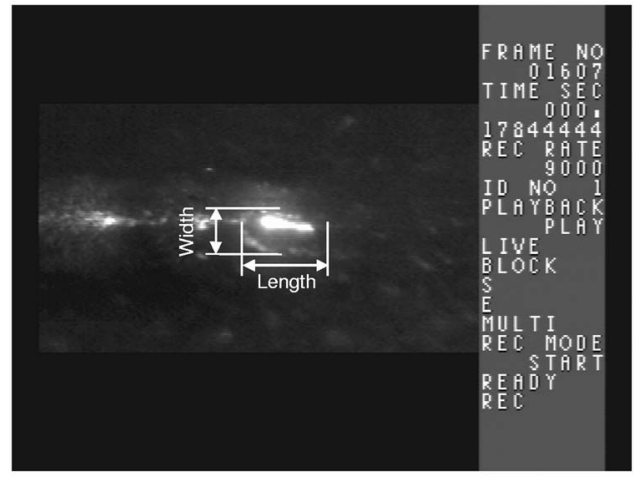

FIG. 14. Velocity field with a laser power of $600 \mathrm{~W}$.

a $63.5 \mathrm{~mm}$ focal lens, and finally dispersed by a diffuser plate for a better resolution of the images. Images are acquired through the high performance image grabber card and digitally recorded with an image analysis software.

Figure 14 shows the picture of the molten pool during the laser cladding with a $600 \mathrm{~W}$ laser beam. The images were taken from a high speed camera at 9000 frames/s recording rate. The images were calibrated to make the pixel coordinates map to real world coordinates. MATROX INSPECTOR $\AA$, an image analysis software package, was used to calibrate and measure the recoded images. To calibrate, a thin paper with a $0.5 \times 0.5 \mathrm{~mm}^{2}$ grid system is attached to a steel substrate, which is identical in thickness to the cladding specimen. Images are acquired under the same experimental configurations and calibrated by the software.

Figure 15 shows the length (in the $X$ direction) and width (in the $Y$ direction)of the molten pool obtained from both experimental and numerical results with the same processing parameters. The trend of the numerical results matches that of the experimental results reasonably. The maximum divergence between the experimental and numerical results is $22 \%$. Compared with the experimental results, the size of the molten pool obtained from numerical results is more sensitive to laser power input, which causes an overestimation at higher power $(700 \mathrm{~W})$ and an underestimation at lower power $(500 \mathrm{~W})$. The discrepancy may be caused by the differences of the real material properties, processing conditions (shielding gas and powder delivery gas), and

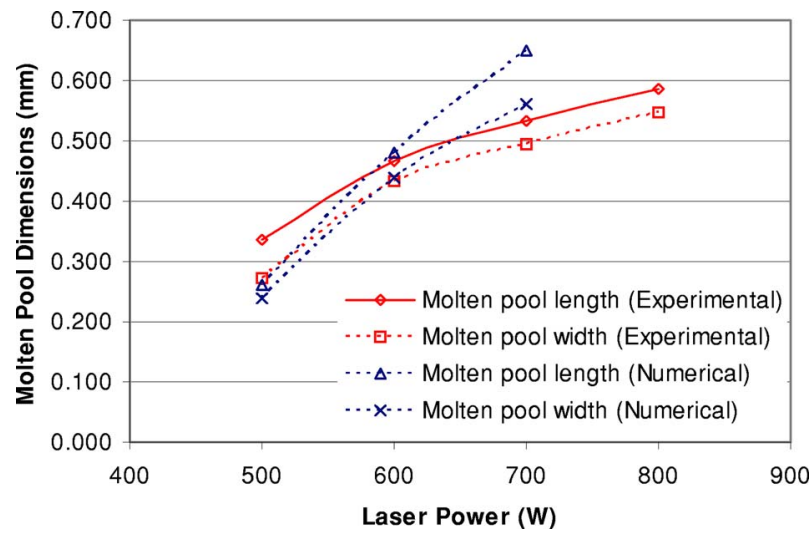

FIG. 15. Comparison of the experimental and numerical results of molten pool dimensions (maximum divergence of $22 \%$ ). 


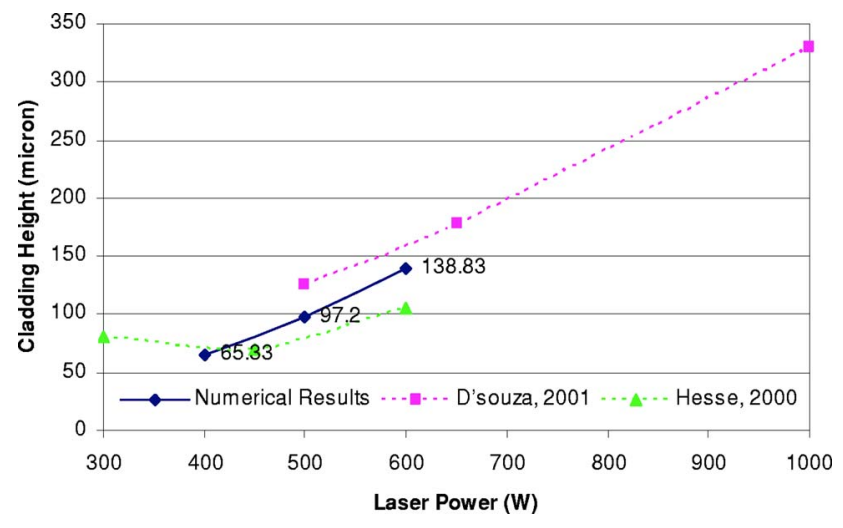

FIG. 16. The experimental and numerical results of H13 single track cladding height.

those used in the modeling. The single track cladding height results are also compared with the experimental data of H13 tool steel from previous works in the CLAIM laboratory at the University of Michigan, ${ }^{24,25}$ as shown in Fig. 16. Again, one observes similar increasing trends of bead height with increasing laser power. The difference of the absolute bead height value between the numerical and experimental results is up to $32 \%$. This is potentially due to the variance of the powder concentration distribution in the real process from the assumed Gaussian distribution in the model. The real powder capture efficiency may also vary from the model, which assumes that powder velocity has zero component in the horizontal plane and all the powder projecting in the molten pool region are captured.

\section{CONCLUSIONS}

A self-consistent three-dimensional model was developed for a coaxial laser powder cladding process, which simulates heat transfer, phase changes, and fluid flow in the molten pool. Transport equations were solved with a controlled-volume finite difference method. Temperature and fluid velocity were solved in a coupled manner.

Physical phenomena at the $\mathrm{L} / \mathrm{G}$ and $\mathrm{S} / \mathrm{L}$ interfaces were successfully incorporated in the governing equations as boundary conditions, such as the thermocapillary and capillary forces at the $\mathrm{L} / \mathrm{G}$ interface in the momentum equations, Stefan's conditions at the S/L interface in the energy equation, and convection and radiation heat losses at the $\mathrm{L} / \mathrm{G}$ interface in the energy equation.

Level-set method was implemented to track the free surface movement of the melt pool due to the powder addition and fluid flow.

A laser-powder interaction model was developed in this study to account for the effects of laser power attenuation and powder temperature rise during the coaxial laser powder cladding process.

Parametric study revealed 7\%-20\% laser power attenuation with a powder flow rate of $6-12 \mathrm{~g} / \mathrm{min}$ in and $7-10 \mathrm{~mm}$ of interaction distance. A fraction of the powder falling within the laser beam may experience phase changes before it reaches the molten pool depending on the laser power and powder velocity.

Temperature and velocity fields reach quasi-steady-state in about $30 \mathrm{~ms}$ with laser power of up to $600 \mathrm{~W}$.

Maximum velocity of $1.12 \mathrm{~m} / \mathrm{s}$ was observed in the melt pool when the flow is fully developed with $600 \mathrm{~W}$ of laser power.

Melt pool length and width increase with increase of laser power. Numerical model predicts higher rate of increase than what was observed experimentally (22\% maximum divergence). However, the trend is similar. Discrepancy may be caused by the high temperature material properties, which are not available in the literature.

Bead height increases with laser power almost linearly, except in the low power range (below $400 \mathrm{~W}$ ) near the required energy threshold.

\section{ACKNOWLEDGMENT}

We wish to acknowledge the support from NSF PREMISE (Grant No.DMI-0225928).

${ }^{1}$ J. Mazumder, J. Choi, K. Nagarathnam, J. Koch, and D. Hetzner, JOM 49, 55 (1997).

${ }^{2}$ R. Vilar, Materials Science Forum 301, 229 (1999).

${ }^{3}$ J. O. Milewski, G. K. Lewis, J. Fonseca, and R. B. Nemec, Mater. Manuf. Processes 15, 247 (2000).

${ }^{4}$ J. Mazumder and H. Qi, Proceedings of SPIE - The International Society for Optical Engineering, Proc. SPIE 5706, 38 (2005).

${ }^{5}$ M. Picasso, C. F. Marsden, J. D. Wagniere, A. Frenk, and M. Rappaz, Mater. Manuf. Processes 25, 281 (1994).

${ }^{6}$ J. Jouvard, D. Grevey, F. Lemoine, and A. Vannes, J. Laser Appl. 9, 43 (1997).

${ }^{7}$ A. F. A. Hoadley and M. Rappaz, Metall. Trans. B 23, 631 (1992).

${ }^{8}$ V. Yevko, C. Park, G. Zak, T. Coyle, and B. Benhabib, Rapid Prototyping J. 4, 168 (1998).

${ }^{9}$ A. F. H. Kaplan and G. Groboth, J. Manuf. Sci. Eng. 123, 609 (2001).

${ }^{10}$ E. Toyserkani, A. Khajepour, and S. Corbin, J. Laser Appl. 15, 153 (2003).

${ }^{11}$ G. Zhao, C. Cho, and J. Kim, Int. J. Mech. Sci. 45, 777 (2003).

${ }^{12}$ Y. Huang, G. Liang, and J. Su, Journal of University of Science and Technology Beijing: Mineral Metallurgy Materials (Eng. Ed) 11, 13 (2004).

${ }^{13}$ W. D. Bennon and F. P. Incropera, Int. J. Heat Mass Transfer 30, 2161 (1987).

${ }^{14}$ S. Osher and J. A. Sethian, J. Comput. Phys. 79, 12 (1988).

${ }^{15}$ D. Adalsteinsson and J. Sethian, J. Comput. Phys. 122, 348 (1995).

${ }^{16}$ T. Chande and J. Mazumder, J. Appl. Phys. 57, 2226 (1985).

${ }^{17}$ J. M. Lin, J. Laser Appl. 12, 28 (2000).

${ }^{18}$ V. R. Voller and C. R. Swaminathan, Numer. Heat Transfer, Part B 19, 175 (1991).

${ }^{19}$ V. R. Voller, A. D. Brent, and C. Prakash, Int. J. Heat Mass Transfer 32, 1719 (1989).

${ }^{20}$ S. Asai and I. Muchi, Trans. Iron Steel Inst. Jpn. 18, 90 (1978).

${ }^{21}$ J. P. Vandoormaal and G. D. Raithby, Numer. Heat Transfer 7, 147 (1984).

${ }^{22}$ H. Gedda, J. Powell, G. Wahlstrom, W. B. Li, H. Engstrom, and C. Magnusson, J. Laser Appl. 14, 78 (2002).

${ }^{23}$ M. Doubenskaia, P. Bertrand, and I. Smurov, Thin Solid Films 453\&454, 477 (2004).

${ }^{24} J$. D'souza, Ms thesis, Mechanical Engineering Department, The University of Michigan, 2001.

${ }^{25}$ T. Hesse, Ms thesis, Mechanical Engineering Department, The University of Michigan, 2000.

${ }^{26} \mathrm{H}$. Ki, P. Mohanty, and J. Mazumder, Metall. Mater. Trans. A 33, 1817 (2002). 